\title{
GPSR+Predict: An Enhancement for GPSR to Make Smart Routing Decision by Anticipating Movement of Vehicles in VANETs
}

\author{
Zineb Squalli Houssaini $^{*}{ }$, Imane Zaimi ${ }^{2}$, Mohammed Oumsis ${ }^{2,3}$, Saïd El Alaoui Ouatik ${ }^{1}$ \\ ${ }^{1}$ IT laboratory and Modeling (LIM) Faculty of Science at Dhar El Mahraz (FSDM) Sidi Mohammed Ben Abdellah University \\ (USMBA), Fez, Morocco. \\ ${ }^{2}$ LRIT, Associated Unit to CNRST (URAC 29) Faculty of Science, Mohammed V University, Rabat, Morocco. \\ ${ }^{3}$ High School of Technology, Mohammed V University, Sale, Morocco.
}

\begin{tabular}{l} 
A R T I C L EI N F O \\
\hline Article history: \\
Received: 06 March, 2017 \\
Accepted: 07 April, 2017 \\
Online: 18 April, 2017 \\
\hline Keywords: \\
VANET \\
GPSR \\
Estimation \\
Routing \\
NS2 \\
VanetMobiSim \\
QoS \\
Highway and urban \\
environments
\end{tabular}

\begin{abstract}
A B S T R A C T
Vehicular ad-hoc networks (VANETs) are still more challenging to overcome even if they have been widely studied during the last decades. The routing mechanism is the essentially relevant issue in this field. Indeed, it must strictly to be adapted to specific and unique characteristics such as the high mobility of the vehicles, the dynamic nature of network topology as well as the high link breakage probability. In this paper, our objective is to improve the greedy perimeter stateless routing protocol (GPSR) as being the most promising position-based mechanism. However, according to the impact of position information on routing decision, our proposed approach defined by GPSR+PRedict protocol ensures that each vehicle estimates its own position for the near future. Afterwards, through extensive experiments, we show the ability of the GPSR+PRedict to overcome the observed problems and to enhance the overall performance of the traditional GPSR approach. The simulations are carried out on both highway and urban scenarios by using NS-2 and VanetMobiSim simulators.
\end{abstract}

\section{Introduction}

Vehicular ad-hoc network (VANET) is a new class of mobile ad-hoc network (MANET) that has emerged through the advances of wireless technologies and automotive industry [1]. This application field has become the core of the intelligent transport systems (ITS) in order to enhance road safety and improve comfort of road users [2, 3]. In addition, VANET's main features are [4]: traffic monitoring, traffic control, increasing visibility in dangerous intersections, detection of collisions and real time traffic calculation. Moreover, other applications apart from intelligent transport reside on providing connectivity to network or any communication between vehicles (e.g. games, chats or file exchange).

VANET behaves distinctly when compared to the other infrastructureless networks. Indeed, it is distinguished by

\footnotetext{
${ }^{*}$ Zineb Squalli Houssaini, Faculty of science at dhar el mahraz (FSDM) sidi mohammed ben abdellah university (USMBA), Fez, Morocco, Contact No: +212676468098 \& Email: zinebsqualli@gmail.com.
}

specific and unique characteristics, especially, the huge mobility of the vehicles and the instability of network topology [5-7]. Consequently, a series of constraints and challenges including security, quality of service (QoS), and routing mechanism should be raised to meet the needs of aforementioned VANET features [8]. For that reason, different proposals and research studies are booming in order to achieve better results than the previously proposed solutions.

To be part of such network and be able to exchange messages smoothly either between vehicles (vehicle-to-vehicle communication $(\mathrm{V} 2 \mathrm{~V})$ ) or between vehicles and roadside infrastructure (vehicle-to-roadside communication (V2R)) [9], the intelligent vehicle represents the main application. Nevertheless, it must be equipped with some electronic tools namely, wireless communication devices, environmental perception devices (e.g. radars, cameras...), geographic positioning systems (e.g. GPS) [10, 11], and a platform for processing incoming and outgoing information. Figure 1 shows some of these intrinsic equipment $[12,13]$. This paper is based 
on purely V2Vad-hoc communication architecture, but it could be easily adapted to V2R communication with only simple changes.

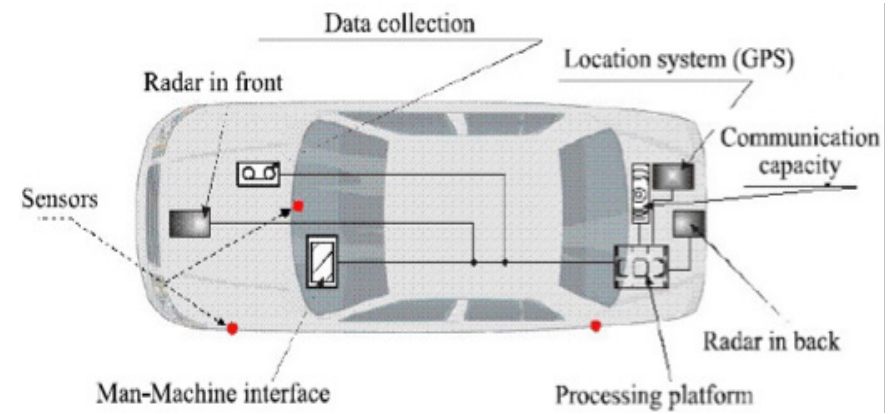

Figure 1: Illustration of different components of an intelligent vehicle [13]

Within this ever-changing vehicular ad-hoc network, messages must be transmitted smoothly from a vehicle to another in order to reach their final destinations. Hence, a routing protocol should be used to ensure inter-vehicular communications in an efficient manner. Exposed to the difficult constraints of vehicular networks, the obvious question is how to ensure routing with a satisfying quality of service (i.e., short delay, minimum lost packets and low overhead).

VANET routing protocols history starts with MANET protocols [14-18]. Unfortunately, these latter cannot achieve a good performance when they are used directly in high challenging environment like VANET. Therefore, many researches focus on adapting these protocols in order to be suitable for VANETs [19-22]. The geographical routing protocols [23-25] are considered as the most stable and functional schemes for VANET's large-scale areas compared to the topology-based routing protocols [26]. The Greedy Perimeter Stateless Routing (GPSR) is a typical example of protocols based on geographical location[27], where each node uses the current information of its position, the position of its neighbors and that of the final destination to make the routing decision.

Considering three significant points, namely, 1) the interest that presents the location's information and vehicles' movement on the both vehicular networks and geographic protocols, 2) the unemployment of amount information, namely, speed and direction given by GPS, 3) the problems observed in geographic protocols as link breakage, it would be advantageous to exploit all these information together to estimate future positions and to consider them during decision process of routing protocols.

In this context, a new geographical routing protocol based on GPSR is proposed. The improvement is mainly defined by using supplementary information to estimate the location of vehicles in the near future. This estimated future position will be included in the periodic message (hello packet) in order to be taken into account in the decision of the best next hop.

The rest of the paper is organized as follows: Section 2 summarizes the related works of routing protocols over VANETs. In Section 3, we provide a brief overview of GPSR protocol. Section 4 describes fully the design of our proposed GPSR+PRedict routing protocol. Simulation settings are presented in section 5, while section 6 shows the effectiveness of GPSR+PRedict via simulation experiments. Finally, the section 7 concludes the study and provides a number of recommendations.

\section{Related Work}

Actually, there are a lot of studies that are interested to the MANET routing protocols adjustment for being used in VANET environments effectively [28]. Additionally, most of researchers are gathering on the point that the geographical routing protocols are the most suitable for vehicular network [26, 29, 30]. Hence, our attention was directed towards one of the protocols belonging to this class: the famous GPSR protocol. GPSR protocol selects the shortest possible route to destination; however, it may suffer from a higher packet error rate due to the poor link quality or the high break link probability.

To this end, many improvements have been recommended. The authors of [31] proposed the cross-layer weighted positionbased routing (CLWPR) to improve the efficiency of geographical routing protocol in vehicular network. The proposed algorithm utilizes information about link layer quality and positioning from navigation to anticipate the characteristics of an urban environment. Compared to the default GPSR, CLWPR demonstrates significantly better performance in terms of packet delivery ratio and end-to-end delay metrics. In the same context, authors of [32], designed an enhanced GPSR protocol and presented its performance for both urban and highway scenarios. The novelty resides on the fact that they used weight calculation to select best neighbor (best next hop). To compute this weight, they used location and velocity information as well as link quality metrics extracted during the hello messages receipt to produce routes that improve the performance of the network. Their proposed protocol achieves higher packet delivery ratio for the network, lower end-to-end delay, while keeping the energy consumption at the same low levels of GPSR. Furthermore, a grid-based predictive geographical routing protocol (GPGR) has been proposed by the researchers of [33]. Indeed, they used a map data to generate the road grid and to predict the moving position during the relay node selection process. Thus, it was noticed that the GPGR protocol reduces the possibility of link breakage and avoids falling on local maximum situation in urban scenarios. Moreover, authors of [33] suggested a new concept based on vehicles movement prediction. In fact, they estimated the Link Stability (LS) for each neighboring vehicle before selecting the next hop for data forwarding/sending. In this way, movement prediction-based routing (MOPR) protocol determines the most stable communication link of the network in terms of communication lifetime with respect to the movement of vehicles. Afterward, they selected the most stable route from the source till the destination.

To the best of our knowledge, all these approaches have been beneficial in most scenarios, but they concentrate all the required calculation on the forwarder during best next hop process or at the moment of receiving beacon packets, which can affect desperately the network's performance. In addition, in most of these aforementioned enhancements, there is a radical change in the process of choosing the best next hop of the default GPSR using more complicated calculations.

For that reason, we propose in our study to filling the observed gaps and to enhance even more the routing process while retaining the basic mechanism of GPSR protocol. Thus, our new GPSR+PRedict routing protocol does not include direction and speed knowledge in the hello packet, but it includes the estimated future position calculated according to this knowledge. Thereby, each vehicle is able to anticipate the state of its neighborhood in the near future. Our idea is directed regarding both highway and urban areas. 


\section{Overview of GPSR}

GPSR was originally created for MANET, but quickly adapted to VANET [27]. It is one of the most known examples that approve the concept of position based routing. GPSR protocol switches between two forwarding strategies depending on the situation of communicating nodes. Usually, when node needs to send packets, it uses the first forwarding strategy defined by greedy forwarding strategy (GFS). In case of failure (i.e., there is no neighbor with least distance to the destination except the transmitter node itself), GPSR bypasses the problem by using the second strategy known by perimeter forwarding strategy (PFS). Figure 2 illustrates the behavior of GPSR.

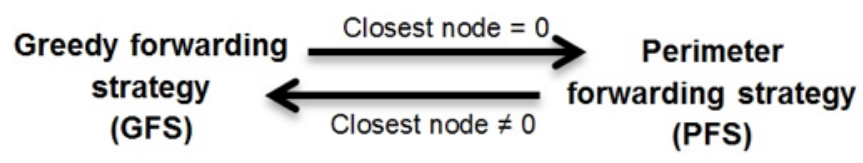

Figure 2: Communication strategies of GPSR

\subsection{Maintain strategy}

In GPSR, before choosing the best path to route a data packet, each node in the network must know its neighbors and their positions. Hence, the nodes flood the network by sending hello packets (beacon packets) containing current position and an identifier. Indeed, each node periodically shares a hello packet with the existing nodes within its own range radio, and simultaneously collects the information sent by these nodes. Due to the beacon packets exchanges, each node can build its own table of neighbors thanks to the collected information, and can maintain it according to the next beacon packets.

\subsection{Routing strategy}

In greedy forwarding strategy (see figure 3), the best next hop is selected based on the optimal path, i.e., it always selects the progressively closer node to the destination. This process is repeated at each intermediate neighbor until the intended destination is reached. With this routing decision, the packet can reach the destination through the optimal path in the aspect of distance. However, sometimes the forwarder vehicle has the shortest distance to destination compared to all its neighbors. In this context, there are two cases. In the first one, the final destination is under the radio range of transmitter vehicle; therefore, packet is forwarded directly. The second case is when the final destination is not accessible in a one hop, so it is impossible to use the GFS. Thus, the PFS is used in order to solve this problem (see figure 4). The PFS algorithm recovers by routing around the perimeter of the region until arriving at a node closer to the destination. At this moment, the GFS takes over.

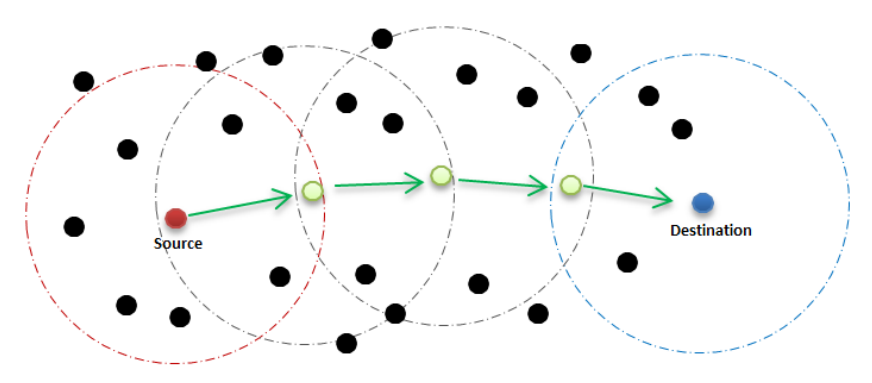

Figure 3: Greedy forwarding packets of GPSR

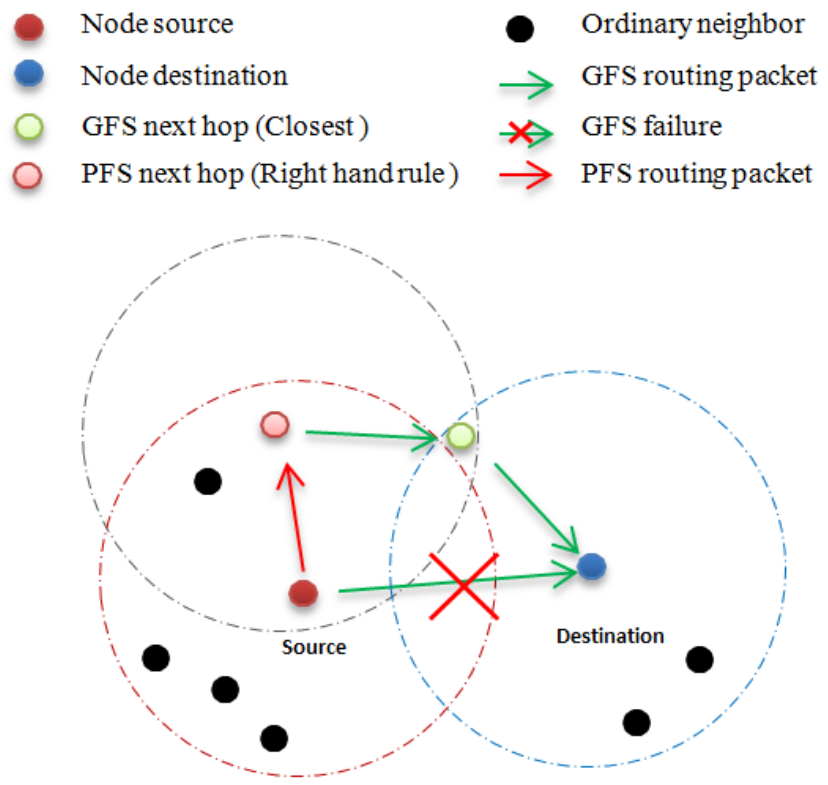

Figure 4: Coping with gap encountered thanks to perimeter forwarding strategy in GPSR

\subsection{Benefits and Drawbacks}

The most important advantage of GPSR, distinctly from the other proactive and reactive protocols, is the fact that the vehicles can get the exact movement information (e.g. geographic position, speed and movement direction) according to a positioning system like GPS or GALILEO. In addition, the one-hop propagated information over the topology, leading to know the geographic position of every single neighbor, minimizes more or less the network overload which is advantageous especially in dense networks. However, regarding the greedy routing strategy, the vehicle often chooses the best next hop in the distance aspect. Nevertheless, without considering speed or moving direction while researching the best next hop, the GPSR protocol could lead to wrong packet forwarding decisions, which may cause packet loss. Moreover, the link breakage problem can also affect the quantity of delivered packets, particularly in highway environment. Actually, when node receives a beacon packet to update its list of neighbors, it computes the best next hop, then it forwards packet. So, during this process, the selected neighbor can be out of the transmission range and the packet cannot reach it because of high network's mobility. In order to solve these problems that lead to performances degradation, we thought that it would be appealing, in terms of QoS, to improve the routing strategy. The enhancements are mainly based on the estimation of the future position of all participating nodes in routing operation. In the next section, a deep description of all proposals is provided.

\section{GPSR+Predict}

\subsection{Motivation}

GPSR+Predict has been conceived on the basis of three significant aspects: First, the importance of node's localization in geographic protocols. Second, the information available in GPS but not exploited by GPSR. Third, the various problems 
encountered, for example, high breakage link probability due to the great mobility with different speeds, bad routing decisions due to opposite directions of vehicles, or inaccuracies in the prediction of the driver's direction at intersections.

In our point of view, it is constructive to propose a new greedy routing mechanism that estimates positions of the vehicles in the near future and takes this information into consideration to make smart routing decision.

\subsection{Prediction the near future position}

With the proposed protocol, each node uses its geographical position, its speed and its direction to estimate its position in a near future. This future position is attached to the hello packets and recalculated at each sent of periodic hello packet. In this way, node continuously has a prediction of future state of the network.

Moreover, unlike the basic GPSR, where the sender node simply searches the nearest neighbor to the destination when it needs to forward a data packet, that of the "GPSR+PRedict" searches firstly if the destination is in its list of neighbors. If it is the case, the packet data is sent directly to the destination, if not, then the sender searches the best next hop in terms of distance by using the estimated future position of neighbors. In fact, GPSR+PRedict practices this strategy in order to know the neighbors that will still or not in sender's neighborhood. Thus, it will make a smarter routing decision by avoiding several bad situations such as link breakage.

The choice of the prediction model is fundamental because it does not affect only the quality of prediction but also the speed and time of the calculation. In fact, there are different mathematical extrapolation methods with different levels of complexity allowing the estimation of future positions of vehicles [35-37]. For this study, we have opted to use a method based on previous positions, direction and speed. This technique is basic with very little intricacy so that the hello packet size does not involve the network overhead (i.e., it does not incur much bandwidth or computational power).

As mentioned above, this model exploits previous positions $\boldsymbol{X}_{\boldsymbol{t} \mathbf{0}}$ and velocity $\overrightarrow{\boldsymbol{V}}\left(\boldsymbol{X}_{\boldsymbol{t} \mathbf{0}}\right)$. The velocity vector is calculated by using the current speed and heading direction of a vehicle (see equations (1) and (2)).

$$
\begin{aligned}
& \overrightarrow{\mathbf{V}}\left(\mathbf{X}_{\mathbf{t} 0}\right)=\mathbf{d} \mathbf{X}_{\mathbf{t} 0} * \text { Speed } \\
& \overrightarrow{\mathbf{V}}\left(\mathbf{Y}_{\mathbf{t} 0}\right)=\mathbf{d} \mathbf{Y}_{\mathbf{t} 0} * \text { Speed }
\end{aligned}
$$

Where $\mathrm{dX}_{\mathrm{t} 0}$ and $\mathrm{dY}_{\mathrm{t} 0}$ are a unit vector that indicate the direction of the vehicle at time $t_{0}$.

Therefore, the future position from any node can be calculated with the following equations (3) and (4):

$$
\begin{aligned}
& \mathbf{f u t}\left(\mathbf{X}_{\mathbf{t} \mathbf{0}}\right)=\mathbf{X}_{\mathbf{t 0}}+\overrightarrow{\mathbf{V}}\left(\mathbf{X}_{\mathbf{t 0}}\right) * \Delta \mathbf{T} \\
& \mathbf{f u t}\left(\mathbf{Y}_{\mathbf{t 0}}\right)=\mathbf{Y}_{\mathbf{t 0}}+\overrightarrow{\mathbf{V}}\left(\mathbf{Y}_{\mathbf{t 0}}\right) * \Delta \mathbf{T}
\end{aligned}
$$

Where $\Delta \mathrm{T}$ is the time between two hello packets.

\subsection{Optimization}

The highlight is to improve routing mechanism by assisting GPSR protocol to make smarter routing decisions and to better choose the suitable neighbor as next hop while maintaining the basic search process of best next hop. Also, we aim to optimize the calculation processing of GPSR and to not concentrate all the processing on the router of the data packet.

From most existing prediction approaches applied to the geographic protocols, the direction and the speed are added then sent in the hello packets. Hence, the nodes do not predict the future positions only after receiving these hello packets or during the research of the next best hop process, i.e., each node must traverse its entire neighbors list and make the required calculation to estimate the future position of each neighbor. This procedure causes two mainly drawbacks: the first is that each node has a lot of calculations to perform, while the second resides on the fact that the same calculation is repeated by several nodes (shared neighbors). As consequence, this strategy of prediction is not optimal and can affect the performance and the QoS especially in dense networks. We have addressed this problem by proposing the idea that each node in the network exploits its information (position and velocity) and estimates its future position in advance, then includes the result in the hello messages (see table 1). In this way, the calculations will be dispersed over the network and will be performed only once by the concerned node.

Through GPSR+PRedict protocol, when a node broadcasts the beacon packet to the other vehicles within its radio range, each vehicle that receive this hello packet, stores all information in its neighbors list. Therefore, the current and the future positions information are available to be used while computing best next hop. The illustration of both hello packet and neighbors list used in GPSR+PRedict is shown respectively in tables 1 and 2 taking the node 1 at time $t_{0}$ as example.

Table 1: Format of hello packet

\begin{tabular}{|c|c|c|c|c|}
\hline ID node & \multicolumn{2}{|c|}{$\begin{array}{c}\text { Geo-coordinates } \\
\text { of node }\end{array}$} & \multicolumn{2}{|c|}{$\begin{array}{c}\text { Future position estimated } \\
\text { of node }\end{array}$} \\
\hline 1 & $X_{t 0}$ & $Y_{t 0}$ & fut $\left(X_{t 0}\right)$ & $f u t\left(Y_{t 0}\right)$ \\
\hline
\end{tabular}

Table 2: Format of neighbors list

\begin{tabular}{|c|c|c|c|c|}
\hline $\begin{array}{c}\text { ID } \\
\text { neighbors }\end{array}$ & \multicolumn{2}{|c|}{$\begin{array}{c}\text { Geo-coordinates } \\
\text { of neighbors }\end{array}$} & \multicolumn{2}{|c|}{$\begin{array}{c}\text { Future position estimated of } \\
\text { neighbors }\end{array}$} \\
\hline 1 & $X_{n 1 \_t 0}$ & $Y_{n 1 \_t 0}$ & fut $\left(X_{n 1 \_t 0}\right)$ & fut $\left(Y_{n 1 \_t 0}\right)$ \\
\hline
\end{tabular}

Figure 5 summarizes all that has been explained concerning our improved greedy routing strategy. It represents the sending process of the hello packets and that of the data packets as well. 


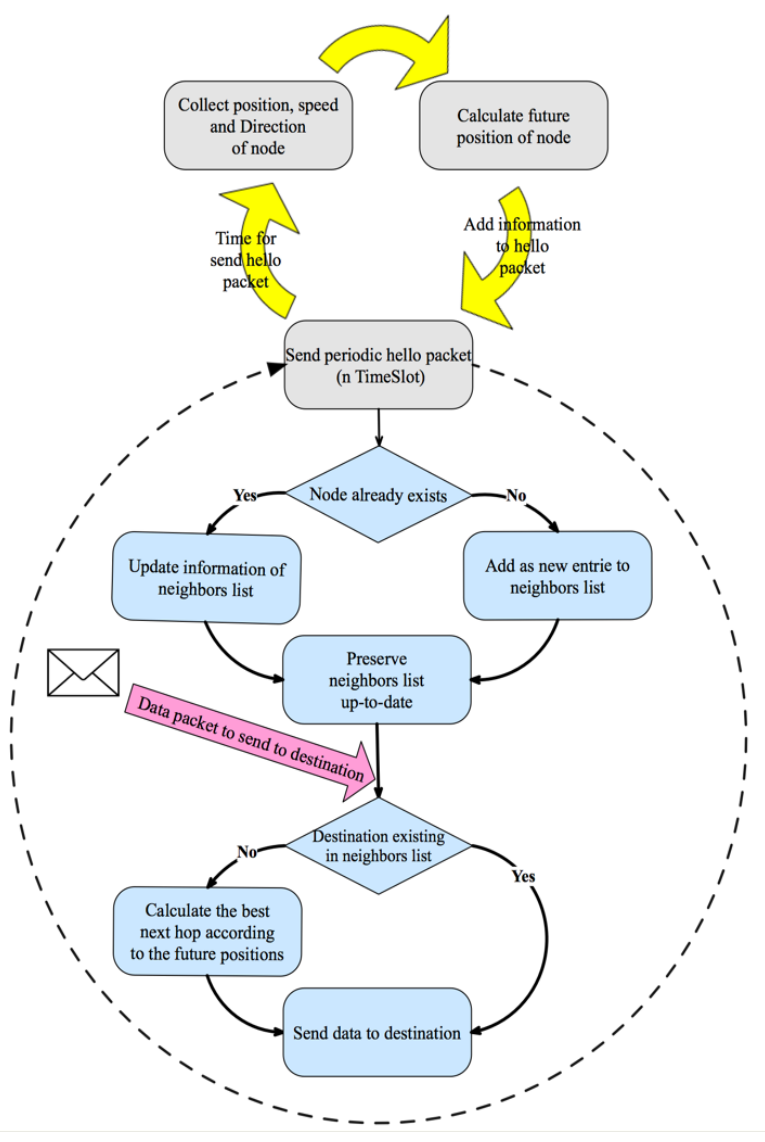

Figure 5: Greedy strategy of new GPSR+PRedict protocol

Our interest to improve the GPSR's routing mechanism is clearly motivated by the three cases presented respectively in figures 6,7 and 8 . It should be noticed that the default GPSR routing mechanism forms the red route (red arrows) where there is often breakage links, while the GPSR+PRedict routing mechanism forms the optimal green route (green arrows).

From figure 6, according to the default greedy strategy, the source node chooses the node A as the next hop since it is the closest one to the final destination. Nevertheless, the packet will be lost since the direction of $\mathrm{A}$ is different from that of the destination. The proposed mechanism forms the green route and avoids the problem of opposite directions that will occur in the red route in a very short amount of time.

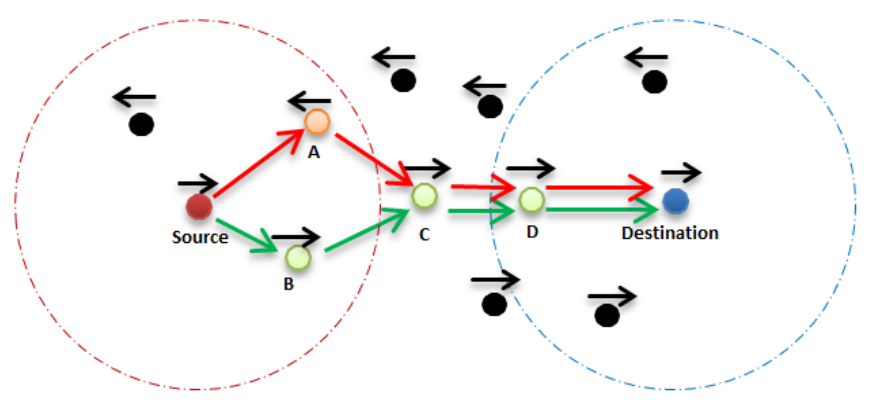

Figure 6: Problem of opposite direction in GPSR

Another problem is overcome as shown in figure 7: the problem of out of the radio range. In fact, according to the default GPSR forwarding process, when the highly mobile node marked by $\mathrm{A}$ is chosen as the next hop to send data packet to the final destination, it will be out of the range before the starting of transmission. In regards to GPSR+PRedict, it anticipates link breakage problem, which certainly will occur, and avoids it by selecting the node B as the next hop.

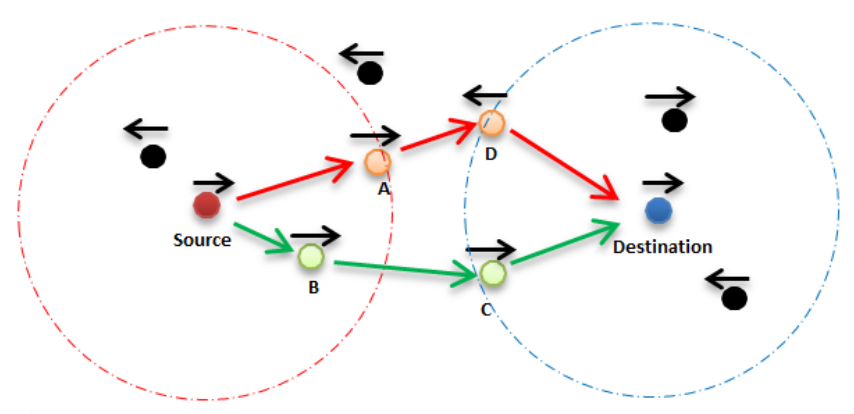

Figure 7: Problem of out of radio range in GPSR

In figure 8 , considering that the vehicle $\mathrm{B}$ is much faster than $A$, so the vehicle $B$ will exceed the vehicle A in a very short period of time. With GPSR+PRedict forwarding process, the source expects this situation since it has a future position estimated of its neighbors A and B. Consequently, the source chooses the node B as next hop instead of node A in case of classical GPSR. Thus, the speed problem is bypassed.

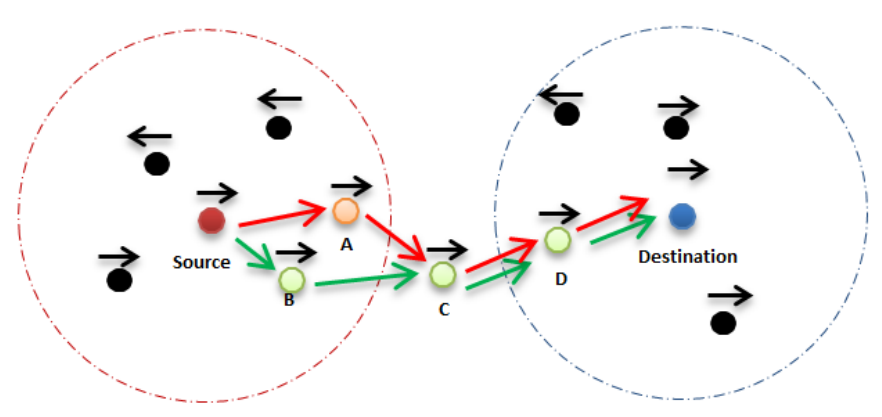

Figure 8: Problem of speed in GPSR

\section{Simulations settings}

\subsection{Parameters of simulation}

In the literature, various tools have been implemented to perform experimental studies. In this paper, we used NS-2 software which is the most employed simulator to study the protocol's performance [38]. Additionally, VanetMobiSim is used as a realistic mobility traffic generator [39, 40], where vehicles travel according to an Intelligent Driver Model with Lane Changes (IDM-LC). VanetMobiSim simulator is considered as the most efficient mobility modeling framework. Indeed, itis widely used to simulate realistic automotive motions at a macroscopic and microscopic level. Here, we have used this software to generate both of realistic mobility models and realistic urban/highway traffic environments. The output mobility trace obtained from VanetMobiSim is directly used as input in NS-2.

In order to evaluate the effectiveness of our enhancement, we have opted to compare our GPSR+PRedict and the default GPSR protocol in highway and urban scenarios since they are two 
Z. Squalli Houssaini et. al. /Advances in Science, Technology and Engineering Systems Journal Vol. 2, No. 3,137-146 (2017) different milieus with a very distinct infrastructures and traffic rules. Each scenario is designed by varying the number of vehicles from 50 to 350 vehicles and keeping data traffic sources number at15 connections. The "TwoRayGround" model is employed as propagation model. The protocol MAC sets according to the IEEE $802.11 \mathrm{p}$ standard, and operates at $5.9 \mathrm{GHz}$ on a $10 \mathrm{MHz}$ control channel $(\mathrm{CCH})$. The PHY data rate is configured to $6 \mathrm{Mbps}$, which is the minimum value to safe the communication [41]. The couples' source/destination were randomly selected and all nodes exchange beacon packets every 5 seconds (i.e., hello period) according to those protocols. Table 3 shows the common configuration parameters for the following set of experiments.

Table 3: Network parameters for both highway and urban scenarios

\begin{tabular}{|l|c|}
\hline \multicolumn{2}{|c|}{ NS2.33 network simulator } \\
\hline \multicolumn{1}{|c|}{ Parameter } & value \\
\hline MAC Layer & $802.11 \mathrm{p}$ \\
\hline Propagation Model & Two-Ray-Ground \\
\hline Radio range & CBR/UDP \\
\hline Traffic model & $5 \mathrm{~s}$ \\
\hline Hello Period & $50 \mathrm{~s}$ \\
\hline Hello Timeout & $500 \mathrm{~s}$ \\
\hline Time Simulation & $1 \mathrm{pkt} / \mathrm{s}$ \\
\hline Packet Interval & 512 octets \\
\hline Packet Size & 15 connections \\
\hline Number of traffic & $50,100,150,200,250,300$ and 350 \\
\hline Number of vehicles & GPSR+PRedict and GPSR \\
\hline Routing Protocols & \\
\hline
\end{tabular}

\subsubsection{Urban scenario}

For urban scenario, a space of $1000 * 1000$ meters $^{2}$ is randomly generated. It consists of various intersections, twoway road, and different density of vehicles in motion with variation speed between 20 and $60 \mathrm{~km} / \mathrm{h}$. Figure 9 illustrates the urban topology used for our experiments, while table 4 shows the parameters of the mobility scenario.

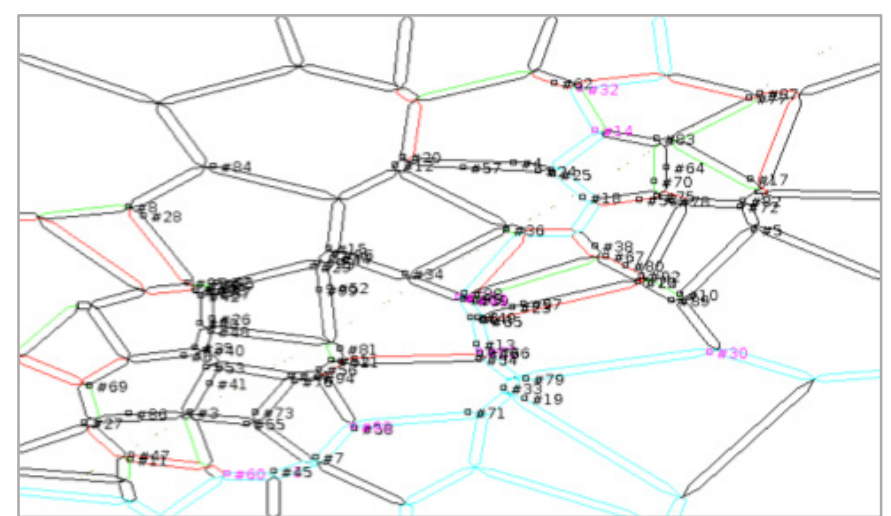

Figure 9: Urban topology randomly generated by VanetMobiSim

Table 4: Urban mobility model parameters

\begin{tabular}{|l|c|}
\hline \multicolumn{2}{|c|}{ VanetMobiSim mobility generator: urban scenario } \\
\hline Parameter & value \\
\hline Space area & IDM-LC \\
\hline Vehicle speed & $1000 * 1000$ meters $^{2}$ \\
\hline Traffic light & 20 to $60 \mathrm{~km} / \mathrm{h}$ \\
\hline Number of lanes per direction & 10 \\
\hline
\end{tabular}

\subsubsection{Highway scenario}

In this subsection, a highway scenario over $700 * 5000$ meters $^{2}$ is randomly generated. It consists of two-way road, i.e., two lanes per direction, without any traffic lights. Different density of vehicles is driving at speeds varying between 80 and $120 \mathrm{~km} / \mathrm{h}$. Figure 9 illustrates an example of the highway topology used for our simulations, while table 5 shows the parameters of the mobility scenario.

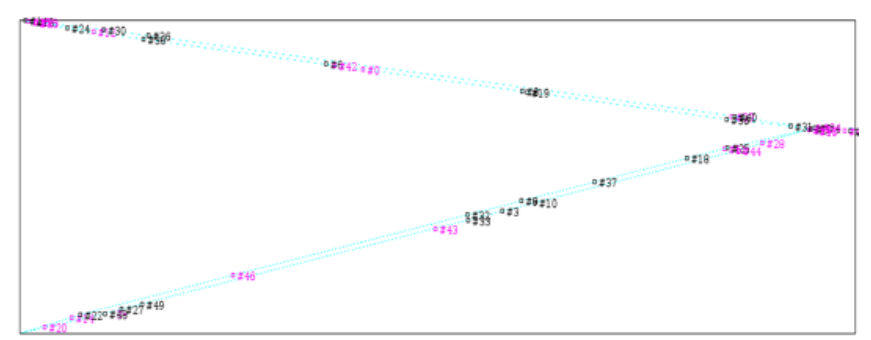

Figure 10: Highway topology randomly generated by VanetMobiSim

Table 5: Highway mobility model parameters

\begin{tabular}{|l|c|}
\hline \multicolumn{2}{|c|}{ VanetMobiSim mobility generator: highway scenario } \\
\hline Parameter & value \\
\hline Mobility model & IDM-LC \\
\hline Space area & $700 * 5000$ meters $^{2}$ \\
\hline Vehicle speed & 80 to $120 \mathrm{~km} / \mathrm{h}$ \\
\hline Traffic light & 0 \\
\hline Number of lanes per direction & 2 \\
\hline
\end{tabular}

\subsection{Metrics}

In order to evaluate and assess the performance of the simulated routing protocols, we use the following metrics:

Packet Delivery Ration (PDR): corresponds to the ratio between the number of packets of data correctly received by the destinations and the number of packets sent by the sources; this latter allows revealing whether a protocol is able to send all outgoing data packets. The final equation of PDR shown in (5):

$$
P D R=\frac{\sum \text { Received Packets }}{\sum \text { Sent Packets }}
$$


Z. Squalli Houssaini et. al. /Advances in Science, Technology and Engineering Systems Journal Vol. 2, No. 3,137-146 (2017)

End-to-End delay average (E2E) is defined as the average time that a data packet takes to travel from the source to the destination vehicle. It represents the main parameter that must be improved during the evaluation of a routing protocol. A good protocol must have an end-to-end delay average as low as possible. Its calculation is as follows in equation (6):

$$
\text { E2E }=\frac{\text { PacketTransmissionTime }}{\sum \text { Received Packets }}
$$

Throughput: it measures the flux or the quantity of data traveling over a communication channel per unit time, usually expressed in bits per second or packets per second. We use the following equation (7) to calculate the average throughput:

$$
\text { Throughput }=\frac{\text { Size of ReceivedPacket }}{\text { TimeReception-TimeSending }}(7)
$$

Route-costs is calculated by dividing the total number of bytes of the routing packets that (includes forwarded routing packets and control packets as well) and the total amount of the received data (see equation (8)).

$$
\text { Routecosts }=\frac{\sum \text { Size of TransmittedRoutingPackets }}{\sum \text { Size of ReceivedDataPackets }}(8)
$$

Normalized routing Load (NRL) defined as the total number of routing packets transmitted per data packet. It calculated by dividing the total sum of routing packets that sent (includes forwarded routing packets as well) by the total number of data packets received as follows in below equation (9):

$$
N R L=\frac{\sum \text { TransmittedRoutingPackets }}{\sum \text { ReceivedDataPackets }}
$$

\section{Simulation Experiments}

Initially, the objective of our experiments is to evaluate the effectiveness of our improved protocol, taking into account the impact of node density regarding routing quality of service. Therefore, we varied the number of vehicles to 50, 100, 150, 200, 250, 300 and 350 respectively, while keeping number of connections fixed at 15 connections. In order to guarantee the credibility and reliability of the experimental results, we respectively made 10 time experiments during 500 seconds for each simulation. The simulations result in both highway and urban are presented in figures 11 to 20 , in which we illustrate the packet delivery ratio, the average end-to-end delay, the throughput, the route-costs and the normalized routing load for GPSR+PRedict and default GPSR.

In figures 11 and 12, default GPSR seems to have worst performance in both scenarios, while our proposed GPSR+PRedict gives good values. This is because GPSR+PRedict anticipates in advance the changes of vehicles localizations. Thus, it avoids the problems caused by the high mobility of the vehicles and the dynamic nature of the topology. In the highway scenario, our approach remarkably improves the PDR compared to the default GPSR since the GPSR mismanages the very high mobility of vehicles.

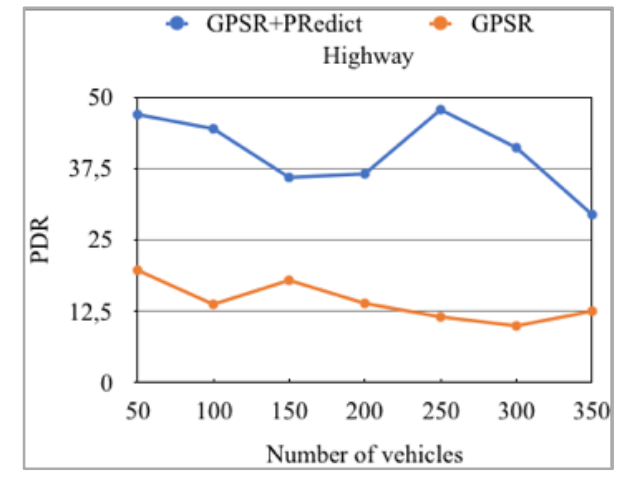

Figure 11: Packet Delivery ratio VS nodes density in highway scenario

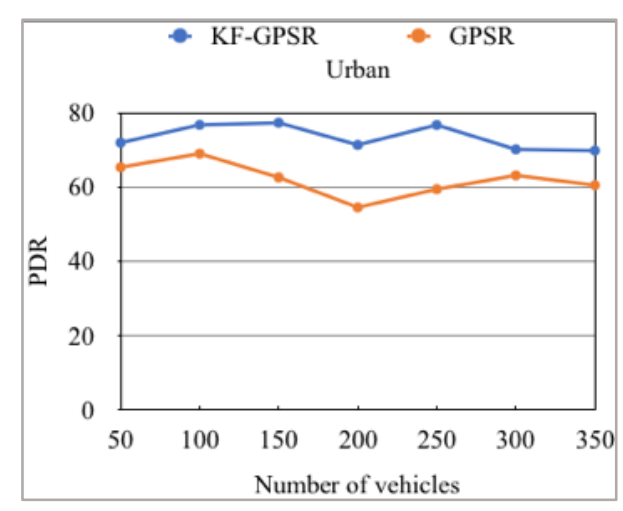

Figure 12: Packet Delivery ratio VS nodes density in urban scenario

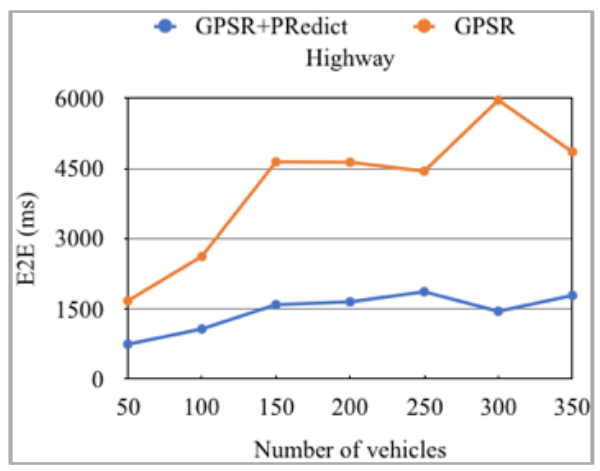

Figure 13: End-to-end delay VS nodes density in highway scenario

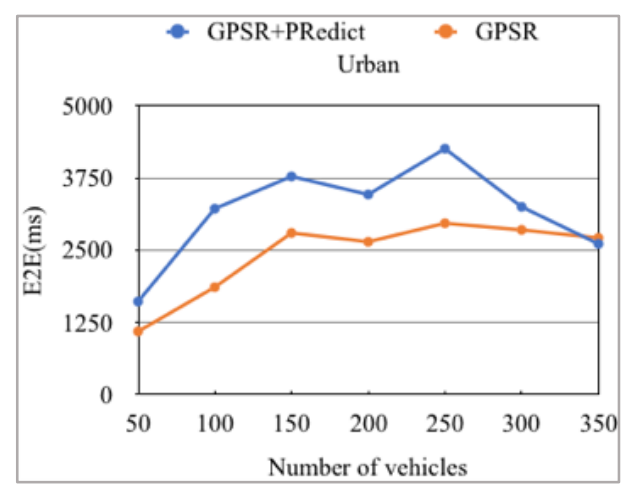

Figure 14: End-to-end delay VS nodes density in urban scenario 
Z. Squalli Houssaini et. al. /Advances in Science, Technology and Engineering Systems Journal Vol. 2, No. 3,137-146 (2017)

In figure 13, regarding the highway scenario, it can be noticed that the classical GPSR has the longer average end-toend delay due to the outdated location information that results very probably data loss. While, GPSR+PRedict decreases Endto-end delay since it avoids the wrong routing decisions by using future position estimated, hence, the routed data packet will have less chance to be lost. In contrast to the urban scenario (see figure 14), we note that our enhanced protocol has the worst end-to-end delay. This is probably because of two things: its higher PDR compared to the default GPSR, and the mobility of vehicles, which is relatively inferior in this environment than in the highway.

Figures 15 and 16 present the flow in function of density. Both figures show that our improved GPSR+PRedict has higher flow than default GPSR. This can be explained by the fact of the good receipt of data packets. As we see, the performance of default GPSR is very low compared to our approach especially in the highway scenario. This is probably because of these bad routing decisions since it does not take into account the high mobility of vehicles, and this is clearly seen from the significant drop shown in figure 16.

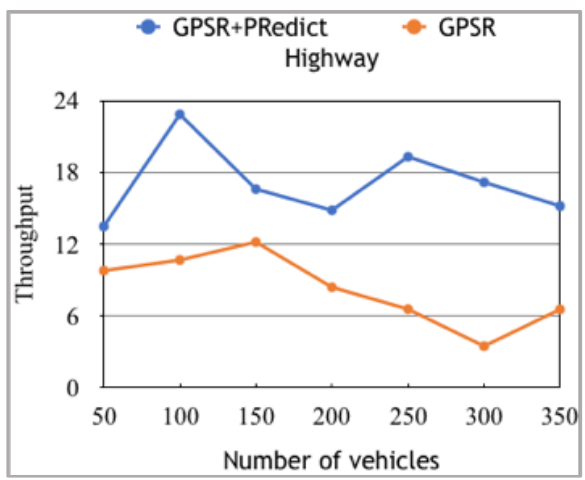

Figure 15: Throughput VS nodes density in highway scenario

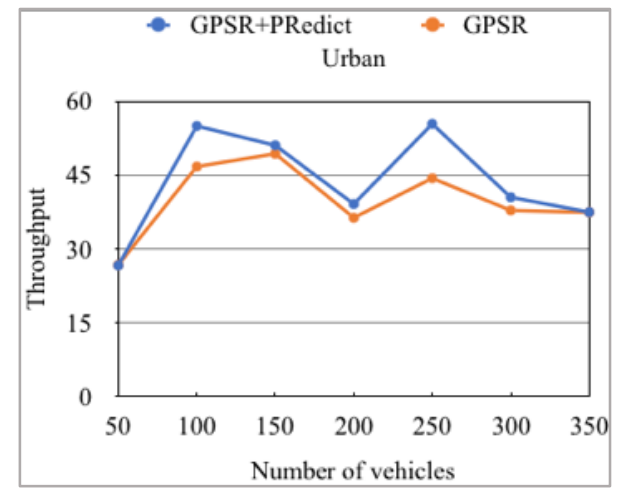

Figure 16: Throughput VS nodes density in urban scenario

Figures 17 and 18 illustrate the cost of routing according to the number of moving vehicles respectively in both highway and urban scenarios. The simulation results show that GPSR+PRedict guarantees low cost of routing compared to the default GPSR in highway scenario (see figure 17). The improved protocol suffers less from link breakages, which conduct to less packet loss and less routing cost. As for results of urban scenario presented in figure 18, GPSR+PRedict has a very slight rise in cost of routing compared with default GPSR. These values can be justified by the high PDR of GPSR+PRedict in the same scenario.

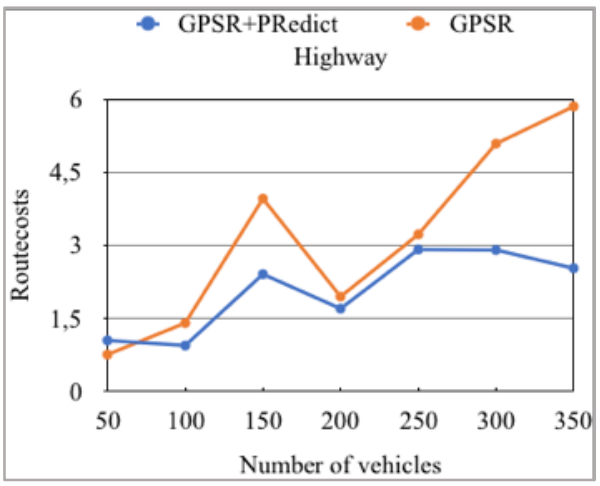

Figure 17: Route-cost VS nodes density in highway scenario

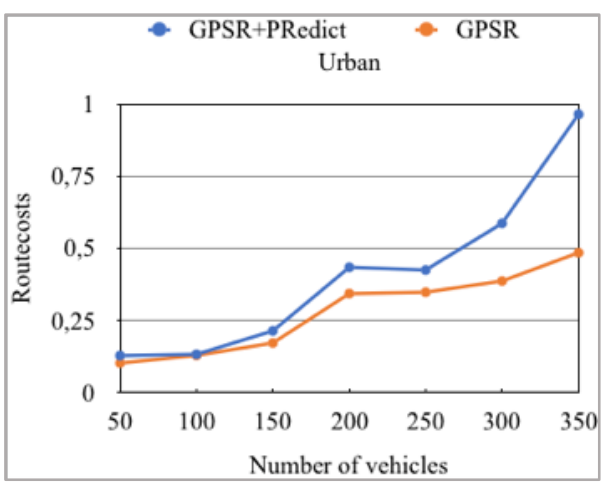

Figure 18: Route-cost VS nodes density in urban scenario

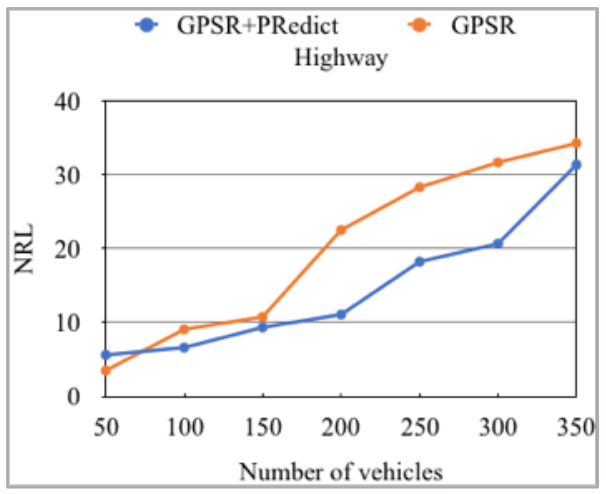

Figure 19: Normalized routing load VS nodes density in highway scenario

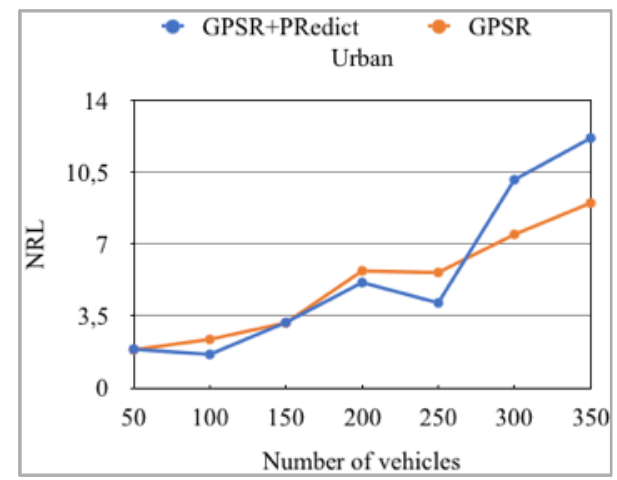

Figure 20: Normalized routing load VS nodes density in urban scenario 


\section{Z. Squalli Houssaini et. al. /Advances in Science, Technology and Engineering Systems Journal Vol. 2, No. 3,137-146 (2017)}

In regards of density, the normalized routing load increases when there are more router packets in the network. As shown in figures 19 and 20, the NRL proportionally increases with the number of vehicles. In The highway environment (figure 19) the performance evaluation shows that GPSR+PRedict outperforms GPSR in term of the normalized routing overload rate. As for the urban environment, presented in figure 20, our enhanced GPSR+PRedict improves the NRL for almost all the tested cases. In fact, default GPSR marks slight results than the GPSR+PRedict, especially in very dense network.

Regarding comparison of the obtained results for both routing protocols, it is clear that the proposed enhancement GPSR+PRedict practically guarantees better performance in all simulations compared to default GPSR.

The enhancements of GPSR protocol that are made in order to adapt it to the specific characteristics of VANET, have shown their gains especially in highway environment where mobility and topology are instable. Indeed, we notice a significant progress with all metrics in all cases compared to default GPSR. Meanwhile, in urban environment where mobility and topology are relatively less dynamic, our approach improves quietly packet delivery ratio, flow and normalized routing load. However, for average end-to-end delay and route-costs, the default GPSR is better, but the deficit is quite small and tolerable. Therefore, the proposed enhancement is a strong candidate to implement with GPSR.

\section{Conclusion and Perspectives}

In this paper, a new routing approach is developed in order to guarantee a high quality of service in VANET areas. The novelty resides on the simplicity of design and identity preserved of the original GPSR protocol. In fact, GPSR+Predict routing protocol exploits in a simple and optimized way node movement information, namely the position, the direction and the speed, to estimate vehicles localization in the near future. This predicted future position will be included in the hello packet and diffused periodically among neighbors, hence, it will greatly contribute to make a smart routing decision and to better select the appropriate next hop.

Our simulations using different frameworks (NS-2, VanetMobiSim) are directed respectively in both highway and urban environments with taking into account the impact of density. The results show that GPSR+PRedict has the ability to preserve and to increase the performance of the vehicular network. Moreover, it indicates that the proposed technique avoids relatively various problems resulting from the delicate properties of this type of networks. Indeed, it achieves a higher packet delivery ratio, a low end-to-end delay, a low routing cost, a low routing overhead and a high throughput, practically in all scenarios.

For future work, we plan to enhance the accuracy of prediction to be more near to reality, and to carry out more enhancement considering QoS on large environments. Furthermore, it should be noted that the proposed approach is appropriate for constant bit rate traffic (CBR); we plan to extend the approach to other types of applications, for example, streaming in multimedia application.

\section{Conflict of Interest}

The authors declare no conflict of interest.

\section{References}

[1] Lee, Kevin C., Uichin Lee, and Mario Gerla. "Survey of routing protocols in vehicular ad hoc networks." Advances in vehicular adhoc networks: Developments and challenges (2010): 149-170.

[2] Ferdinands, A. "Intelligent transport systems and road safety." INSURANCE COMMISSION OF WESTERN AUSTRALIA CONFERENCE ON ROAD SAFETY, 1999, PERTH, WESTERN AUSTRALIA. 1999.

[3] Rumar, K., et al. "Intelligent transport systems and road safety." (1999).

[4] Li, Fan, and Yu Wang. "Routing in vehicular ad hoc networks: A survey." IEEE Vehicular technology magazine 2.2 -2007.

[5] Berbineau, Marion, et al. "Communication technologies for vehicles." NewYork: Springer. doi 10.1007 -2013-: 978-3

[6] Xin Wang, editor. Mobile Ad-Hoc Networks: Applications. INTECH, January 30, 2011. DOI: 10.5772/882

[7] Moustafa, Hassnaa, and Yan Zhang. Vehicular networks: techniques, standards, and applications. Auerbach publications, 2009.

[8] Blum, Jeremy J., Azim Eskandarian, and Lance J. Hoffman. "Challenges of intervehicle ad hoc networks." IEEE transactions on intelligent transportation systems 5.4 -2004-: 347-351.

[9] WAN, Jiafu, YAN, Hehua, SUO, Hui, et al. Advances in CyberPhysical Systems Research. TIIS, 2011, vol. 5, no 11, p. 1891-1908.

[10] BH-Wellenhof, H. Lichtenegger, and J. Collins. "Global Positioning System: theory and practice." 4th ed., Springer-Verlag, 1997.

[11] Elliott, D. Kaplan, and J. Hegarty Christopher. "Understanding GPS: principles and applications." Edition Kaplan, 1996.

[12] Kumar, Rakesh, and Mayank Dave. "Mobile agent as an approach to improve qos in vehicular ad hoc network." arXiv preprint arXiv:1108.2095, 2011.

[13] J. P. Hubaux, S. Capkun and Jun Luo, "The security and privacy of smart vehicles," in IEEE Security \& Privacy, vol. 2, no. 3, pp. 4955, May-June 2004. doi: 10.1109/MSP.2004.26

[14] Li, Huaizhi, and Mukesh Singhal. "A scalable routing protocol for ad hoc networks." Vehicular Technology Conference, 2005. VTC 2005-Spring. 2005 IEEE 61st. Vol. 4. IEEE, 2005.

[15] Giruka, Venkata C., and Mukesh Singhal. "A self-healing ondemand geographic path routing protocol for mobile ad-hoc networks." Ad Hoc Networks 5.7 -2007-: 1113-1128.

[16] De, Swades, and Sajal K. Das. "Dynamic multipath routing -DMPR-: An approach to improve resource utilization in networks for realtime traffic." Modeling, Analysis and Simulation of Computer and Telecommunication Systems, 2001. Proceedings. Ninth International Symposium on. IEEE, 2001.

[17] Haas, Zygmunt J., and Marc R. Pearlman. "The performance of query control schemes for the zone routing protocol." IEEE/ACM Transactions on Networking -TON- 9.4 -2001-: 427-438.

[18] Boleng, Jeff, and Tracy Camp. "Adaptive location aided mobile ad hoc network routing." Performance, Computing, and Communications, 2004 IEEE International Conference on. IEEE, 2004.

[19] Saeed Ahmadi Arzil, Majid Hosseinpour Aghdam and M. A. J. Jamali, "Adaptive routing protocol for VANETs in city environments using real-time traffic information," 2010 International Conference on Information, Networking and Automation -ICINA-, Kunming, 2010, pp. V2-132-V2-136. doi: 10.1109/ICINA.2010.5636966

[20] Lochert, Christian, et al. "A routing strategy for vehicular ad hoc networks in city environments." Intelligent Vehicles Symposium, 2003. Proceedings. IEEE. IEEE, 2003.

[21] Viriyasitavat, Wantanee, Fan Bai, and Ozan K. Tonguz. "Dynamics of network connectivity in urban vehicular networks." IEEE Journal on Selected Areas in Communications 29.3 -2011-: 515-533.

[22] Blum, Jeremy J., Azim Eskandarian, and Lance J. Hoffman. "Challenges of intervehicle ad hoc networks." IEEE transactions on intelligent transportation systems 5.4 -2004-: 347-351.

[23] Leontiadis, Ilias, and Cecilia Mascolo. "Geopps: Geographical opportunistic routing for vehicular networks." World of Wireless, Mobile and Multimedia Networks, 2007. WoWMoM 2007. IEEE International Symposium on a. Ieee, 2007. 
Z. Squalli Houssaini et. al. /Advances in Science, Technology and Engineering Systems Journal Vol. 2, No. 3,137-146 (2017)

[24] Mo, Zhaomin, et al. "MURU: A multi-hop routing protocol for urban vehicular ad hoc networks." Mobile and Ubiquitous Systems: Networking \& Services, 2006 Third Annual International Conference on. IEEE, 2006.

[25] Ding, Yong, Chen Wang, and Li Xiao. "A static-node assisted adaptive routing protocol in vehicular networks." Proceedings of the fourth ACM international workshop on Vehicular ad hoc networks. ACM, 2007

[26] Altayeb, Marwa, and Imad Mahgoub. "A survey of vehicular ad hoc networks routing protocols." International Journal of Innovation and Applied Studies 3.3 2013-: 829-846.

[27] Karp, Brad, and Hsiang-Tsung Kung. "GPSR: Greedy perimeter stateless routing for wireless networks." Proceedings of the 6th annual international conference on Mobile computing and networking. ACM, 2000.

[28] Fazio, Peppino, et al. "A new channel assignment scheme for interference-aware routing in vehicular networks." Vehicular Technology Conference -VTC Spring-, 2011 IEEE 73rd. IEEE, 2011.

[29] Liu, J., Wan, J., Wang, Q. et al. TelecommunSyst -2016- 62: 15. doi:10.1007/s11235-015-9979-7

[30] Cho, Kuk-Hyun, and Min-Woo Ryu. "A survey of greedy routing protocols for vehicular ad hoc networks." SmartCR 2.2 -2012-: 125137.

[31] Katsaros, Konstantinos, et al. "CLWPR - a novel cross-layer optimized position based routing protocol for VANETs." Vehicular Networking Conference -VNC-, 2011 IEEE. IEEE, 2011.

[32] Bouras, Christos, Vaggelis Kapoulas, and Enea Tsanai. "A GPSR enhancement mechanism for routing in VANETs." International Conference on Wired/Wireless Internet Communication. Springer International Publishing, 2015.

[33] Cha, Si-Ho, Keun-Wang Lee, and Hyun-Seob Cho. "Grid-based predictive geographical routing for inter-vehicle communication in urban areas." International Journal of Distributed Sensor Networks (2012).

[34] Menouar, Hamid, Massimiliano Lenardi, and FethiFilali. "Movement prediction-based routing -MOPR- concept for positionbased routing in vehicular networks." Vehicular Technology Conference, 2007. VTC-2007 fall. 2007 IEEE 66th. IEEE, 2007.

[35] D. Katsaros and Y. Manolopoulos, "Prediction in wireless networks by Markov chains," in IEEE Wireless Communications, vol. 16, no. 2, pp. 56-64, April 2009. doi: 10.1109/MWC.2009.4907561

[36] Feng, Huifang, et al. "Location prediction of vehicles in VANETs using a Kalman filter." Wireless personal communications 80.2 (2015): 543-559.

[37] Coupelon, Olivier. "Neural network modelling for stock movement prediction A state of the art." Blaise Pascal University (2007).

[38] "The network simulator ns-2," 2010. [Online]. Available :http://www.isi.edu/nsnam/ns.

[39] Härri, Jérôme, et al. "VanetMobiSim: generating realistic mobility patterns for VANETs." Proceedings of the 3rd international workshop on Vehicular ad hoc networks. ACM, 2006.

[40] Harri, J., and Marco Fiore. "VanetMobiSim-Vehicular Ad hoc Network mobility extension to the CanuMobiSim framework." Institute Eurecom Department of Mobile Commu 6904 (2006): 1-19.

[41] Jiang, Daniel; Chen, Qi; Delgrossi, Luca; Optimal data rate selection for vehicle safety communications, Proceedings of the fifth ACM international workshop on Vehicular Inter-NETworking,30-38,2008 